\title{
NUEVOS REGISTROS PARA EL GECKO DE LIMA, Phyllodactylus sentosus (Reptilia, Geckonidae)
}

\section{NEW RECORDS OF THE LIMA GECKO, Phyllodactylus sentosus (Reptilia, Geckonidae)}

\author{
E. Daniel Cossíos Meza ${ }^{1}$ y Javier Icochea ${ }^{2}$
}

\section{Resumen}

El gecko Phyllodactylus sentosus ha sido registrado únicamente en unos pocos sitios arqueológicos de la ciudad de Lima, de donde es endémico, y es considerado una especie en eligro rítico, conociéndose muy poco sobre su distribución y estado de conservación. En la presente publicación se presenta 4 nuevos lugares de registro para esta especie, todos dentro de la ciudad de Lima.

Palabras clave: Phyllodactylus sentosus, geckonidae, distribución, Lima, especies amenazadas, nuevos registros

The gecko Phyllodactylus sentosus has been registered only in few archeological sites in Lima city, from where it is endemic, and is considered a species in critical danger, with scarce knowledge of its distribution and conservation status. In the present publication we present 4 new records for this species, all of them within the city of Lima, Peru.

Key words: Phyllodactylus sentosus, geckonidae, distribution, Lima, threatened species, new records

\section{Introducción}

El gecko de Lima, Phyllodactylus sentosus, fue descrito a partir de 6 ejemplares, siendo la Universidad de San Marcos el único sitio de distribución conocido para la especie en los años setenta (Dixon \& Huey, 1970). Posteriormente se le encontró en los sitios arqueológicos de huaca Pucllana (distrito de Miraflores) y las huacas del Parque de las Leyendas (distrito de San Miguel). Estos tres lugares se encuentran en la ciudad de Lima y son los únicos sitios para los que el gecko de Lima había sido registrado hasta el momento. Icochea (1998) propuso a Phyllodactylus sentosus como especie "En Peligro Crítico”, categoría correspondiente a las especies con mayor amenaza de extinción y en la que fue incluida por el Estado peruano el 2004 (Decreto Supremo $\mathrm{N}^{\circ}$ 034-2004-AG). A pesar de que esta calificación resalta la urgencia de acciones de conservación, la falta de conocimiento sobre las poblaciones y distribución real de la especie dificultan la toma de decisiones para su manejo. En esta publicación se presenta cuatro nuevos lugares de registro para $P$. sentosus.

\section{Métodos}

Entre Julio de 2005 y Mayo de 2006 se realizó una búsqueda de geckos en cuatro sitios arqueológicos dentro de la ciudad de Lima. Cuando fue posible, las búsquedas fueron nocturnas, con ayuda de linternas y consistieron en caminar a lo largo de senderos ya establecidos dentro de los sitios arqueológicos, detectando a los animales allí presentes. Cuando la búsqueda nocturna no fue posible, se realizó una búsqueda diurna levantando escombros, sin dañar los restos arqueológicos. Los animales encontrados fueron identificados siguiendo las descripciones dadas por Dixon \& Huey (1970). Los lugares de distribución conocidos para $P$. sentosus, incluyendo los nuevos registros, se muestran en la Figura 2 y se listan, presentando su ubicación y área total aproximada, en la Tabla 1.

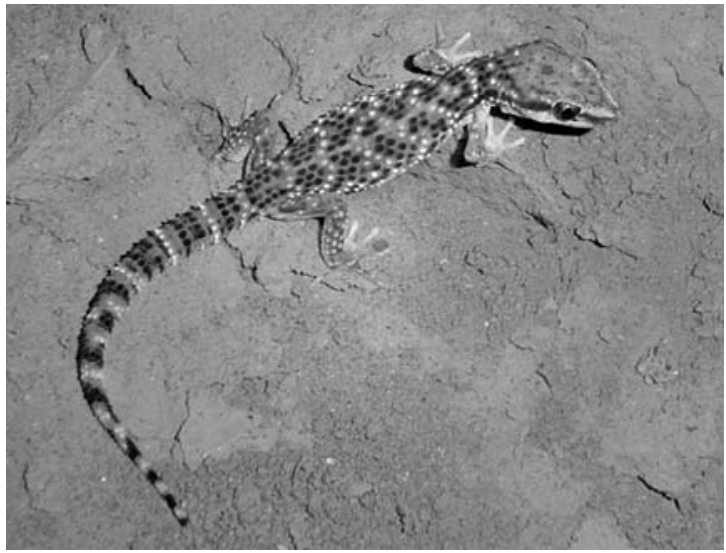

Figura 1. Ejemplar adulto de Phyllodactylus sentosus fotografiado en la Zona Arqueológica de Pachacamac. 
Tabla 1. Lugares de distribución conocidos para $P$. sentosus.

\begin{tabular}{|c|c|c|c|}
\hline Lugar & Dirección & $\begin{array}{l}\text { Coordenadas* y } \\
\text { altitud }\end{array}$ & $\begin{array}{l}\text { Área } \\
\text { Aprox. }\end{array}$ \\
\hline Pucllana & $\begin{array}{l}\text { Gral. Borgoño cdra. } 8 \text { s/n. } \\
\text { Miraflores. }\end{array}$ & $\begin{array}{l}12^{\circ} 06^{\prime} 38.02^{\prime \prime} \mathrm{S} \\
77^{\circ} 01^{\prime} 59.67^{\prime \prime} \mathrm{W}\end{array}$ & $\begin{array}{c}6.07 \\
\text { ha }\end{array}$ \\
\hline $\begin{array}{l}\text { San } \\
\text { Marcos }\end{array}$ & $\begin{array}{l}\text { Ciudad Universitaria. Av. } \\
\text { Venezuela Cuadra } 34 . \\
\text { Lima. }\end{array}$ & $\begin{array}{l}12^{\circ} 03^{\prime} 35.81^{\prime \prime ~ S} \\
77^{\circ} 05^{\prime} 10.57^{\prime \prime} \mathrm{W}\end{array}$ & $\begin{array}{c}6.27 \\
\text { ha }\end{array}$ \\
\hline $\begin{array}{l}\text { Parque de } \\
\text { las } \\
\text { leyendas }\end{array}$ & $\begin{array}{l}\text { Esquina entre Av. De los } \\
\text { Precursores y Av. Parque } \\
\text { de las Leyendas. San } \\
\text { Miguel. }\end{array}$ & $\begin{array}{l}12^{\circ} 04^{\prime} 22.37^{\prime \prime} \mathrm{S} \\
77^{\circ} 05^{\prime} 02.88^{\prime \prime} \mathrm{W} \\
12^{\circ} 04^{\prime} 09.96^{\prime \prime} \mathrm{S} \\
77^{\circ} 05^{\prime} 00.58^{\prime \prime} \mathrm{W}\end{array}$ & $\begin{array}{c}6.10 \\
\text { ha }\end{array}$ \\
\hline $\begin{array}{l}\text { Hualla- } \\
\text { marca }\end{array}$ & $\begin{array}{l}\text { Nicolás de Rivera 201, San } \\
\text { Isidro. }\end{array}$ & $\begin{array}{l}12^{\circ} 05^{\prime} 50.64 " \mathrm{~S} \\
77^{\circ} 02^{\prime} 25.56^{\prime \prime} \mathrm{W}\end{array}$ & $\begin{array}{c}1.58 \\
\text { ha }\end{array}$ \\
\hline $\begin{array}{l}\text { Mateo } \\
\text { Salado }\end{array}$ & $\begin{array}{l}\text { Esquina entre E. García } \\
\text { Rossell y Belisario Sosa } \\
\text { Peláez. Lima. }\end{array}$ & $\begin{array}{l}12^{\circ} 03^{\prime} 59.23^{\prime \prime} \mathrm{S} \\
77^{\circ} 03^{\prime} 49.86^{\prime \prime} \mathrm{W}\end{array}$ & $\begin{array}{c}16.40 \\
\text { ha }\end{array}$ \\
\hline $\begin{array}{l}\text { Pacha- } \\
\text { camac } \\
\text { Puruchu- } \\
\text { co }\end{array}$ & $\begin{array}{l}\text { Antigua Panamericana Sur } \\
\text { km } 31.5 \text { Lurín. } \\
\text { Carretera Central Km } 4.5 \\
\text { Ate. }\end{array}$ & $\begin{array}{l}12^{\circ} 15^{\prime} 31.73^{\prime \prime} \mathrm{S} \\
76^{\circ} 53^{\prime} 58.36^{\prime \prime} \mathrm{W} \\
12^{\circ} 02^{\prime} 58.33^{\prime \prime} \mathrm{S} \\
76^{\circ} 56^{\prime} 07.87^{\prime \prime} \mathrm{W}\end{array}$ & $\begin{array}{l}492.00 \\
\text { ha } \\
\text { Sin } \\
\text { datos }\end{array}$ \\
\hline
\end{tabular}

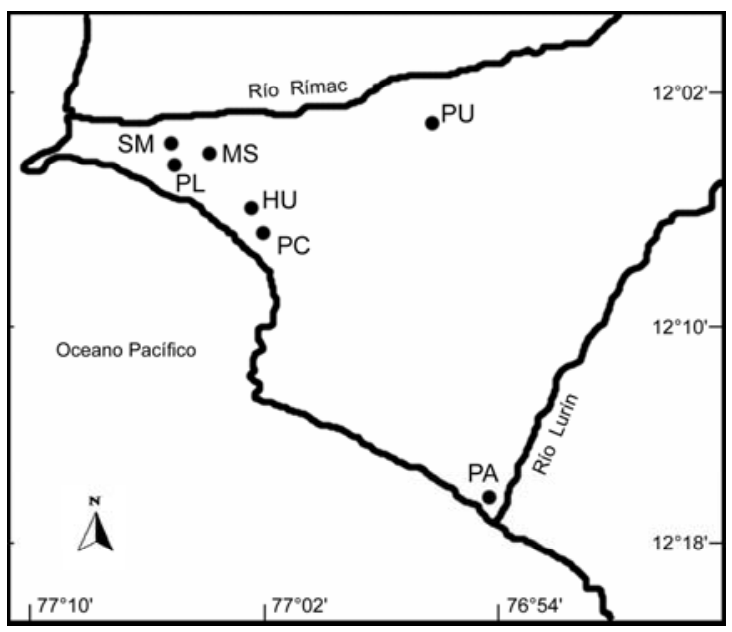

Figura 2. Lugares de registro de $P$. sentosus. $\mathrm{SM}=$ San Marcos, $\mathrm{PL}=$ Parque de las Leyendas, $\mathrm{PC}=$ Pucllana, MS = Mateo Salado, HU = Huallamarca, $\mathrm{PU}$ = Puruchuco, $\mathrm{PA}=$ Pachacamac.

\section{Resultados y discusión}

El primer nuevo registro corresponde a la huaca Huallamarca, ubicada en el distrito de San Isidro, Lima. En este lugar se encontró 8 individuos de $P$. sentosus (6 adultos y 2 juveniles) en 2 horas de búsqueda diurna (evaluación realizada por una sola persona en Julio de 2005). El segundo nuevo registro se hizo en la Zona Arqueológica de Pachacamac, en el distrito de Lurín, Lima (Figura 1), en Enero de 2006. Cabe resaltar que en Pachacamac se repartió la búqueda en dos ambientes: 4 horas/persona (4 personas buscando durante una hora) en el lugar conocido como "peregrinos", que consiste en una zona bastante plana y arenosa, y 4 horas/persona en la "pirámide de Taurichumbi", que presenta principalmente estructuras de adobe y piedras. En el primer lugar se halló 3 individuos adultos de Phyllodactilus microphillus, mientras que en el segundo se encontró un solo individuo, adulto, de $P$. sentosus. El tercer y cuarto registros fueron hechos en Mayo de 2006, en la huaca Mateo Salado (distrito de San Miguel) y en el sitio arqueológico de Puruchuco (distrito de Ate). En Mateo Salado, puesto que no se consiguió autorización para trabajar de noche, se realizó una búsqueda diurna de cuatro horas, por una sola persona, levantando desmonte y se encontró dos individuos adultos de $P$. sentosus. En Puruchuco se llevó a cabo una búsqueda nocturna de 6 horas/persona (3 personas en 2 horas) y se halló dos individuos de $P$. sentosus (un juvenil y un adulto) y uno de $P$. microphillus (adulto).

Una búsqueda realizada por una sola persona en la huaca Pucllana en abril de 2003 arrojó un resultado de 54 individuos de $P$. sentosus en 3 horas (D. Cossíos, datos no publicados), lo que da una idea de la baja densidad de las poblaciones de esta especie en los nuevos lugares de registro, exceptuando Huallamarca. El mayor número de individuos encontrados en Pucllana y Huallamarca (considerando que en este último sitio la búsqueda se hizo de día) podría deberse a que estos sitios arqueológicos, a diferencia de los otros, se encuentran en mantenimiento constante, presentando gran número de intersticios entre los adobes que forman parte de las ruinas, los que podrían ser usados como escondites por los geckos o sus presas.

\section{Conclusiones}

Con los nuevos registros presentados, la distribución conocida de $P$. sentosus se extiende 22 $\mathrm{km}$ hacia el sur-este y continúa restringida a unos pocos lugares arqueológicos situados entre los ríos Rímac y Lurín, en el departamento de Lima. De todos los lugares de registro, Puruchuco es el más alejado de la línea costera $(13.9 \mathrm{~km})$ y el situado a mayor altitud (320 msnm).

Aún considerando los nuevos lugares de registro, la categoría correspondiente a $P$. sentosus, siguiendo los criterios de la UICN (UICN, 2001), continuaría siendo "En Peligro Crítico", debido a poseer un área de ocurrencia estimada menor a 10000 ha, con reducción continua (constatada o deducida) del área de ocurrencia, del área de ocupación, de la superficie o calidad del hábitat y del número de individuos maduros.

Debido a que la Zona Arqueológica de Pachacamac posee un área extensa comparada a las de los otros lugares donde $P$. sentosus ha sido registrado (Tabla 1), dicho lugar puede tener una gran importancia en la conservación de esta especie. Varios autores, sin embargo, señalan marcadas diferencias en 
las preferencias de hábitat de especies parapátricas de geckos (e.g. Huey, 1979; Pianka \& Pianka 1976), por lo que es importante estudiar el uso del espacio por $P$. sentosus y P. microphillus en Pachacamac, así como el tamaño de sus poblaciones dentro de éste y otros sitios para darnos una mejor idea de la importancia de cada lugar.

\section{Agradecimientos}

Un agradecimiento sincero al personal de los lugares arqueológicos visitados, principalmente a Marcelo Saco, director de Pachacamac, Teresa Verástegui, directora de Puruchuco e Isabel Flores, directora de Pucllana, a quienes ayudaron en las búsquedas de campo: Leonardo Maffei, Juan Pablo Nugent y César Nugent -quien detectó los geckos que hallamos en Puruchuco- y, especialmente, a Gino Germaná, quien nos brindó una gran ayuda con los cálculos de distancias y ubicación de coordenadas.

\section{Literatura citada}

Dixon J.R. \& Huey R.B. 1970. Systematics of the lizards of the gekkonid genus Phyllodactylus of mainland South America. Contributions in science, Los Angeles County Museum of Natural History. 192: 1-78.

Icochea J. 1998. Lista roja preliminar de los anfibios y reptiles amenazados del departamento de Lima. Pp:217229, In: Asunción Cano \& Kenneth R. Young (eds). Los pantanos de Villa, Biología y conservación. UNMSM Museo de Historia Natural. Serie de divulgación $\mathrm{N}^{\circ} 11$, 229pp. Lima.

Huey R.B. 1979. Parapatry and niche complementarity of Peruvian desert geckos (Phyllodactylus): the ambiguous role of competition. Oecologia. 38: 249-259.

Pianka E.R. \& Pianka H.D. 1976. Comparative ecology of twelve species of nocturnal lizards (Gekkonidae) in the Western Australian desert. Copeia. 1976: 125-142.

UICN. 2001. Catégories et Critères de l'UICN pour la Liste Rouge : Version 3.1. Commission de la sauvegarde des espèces de l'UICN. UICN, Gland, Suisse et Cambridge, Royaume-Uni. ii .

\footnotetext{
${ }^{1}$ Université de Montréal. Département de Sciences Biologiques. C.P.6126, Succursale centre-ville. Pavillon Marie-Victorin. Montréal QC. H3C 3J7. dcossios@yahoo.com

${ }^{2}$ PO Box 14-219, Lima 14, Perú. anfirep@yahoo.es
} 